\title{
FE II LINE WIDTHS AS TRACERS FOR THE GEOMETRY OF Be STAR ENVELOPES
}

\author{
R. W. HANUSCHIK* \\ Astronomical Institute, Ruhr-Universität, D-44780 Bochum, Germany
}

\section{Motivation}

The geometry of Be star envelopes is not directly observable, apart from those very few cases where interferometry has been successful. This is even more true for the kinematical conditions in these envelopes. An indirect measure of kinematics, density law and geometry can be achieved by comparing line widths of photospheric absorption lines $\left(\Longleftrightarrow v_{*} \sin i\right)$ and circumstellar emission lines $(\Longleftrightarrow v(r) \sin i)$. Hitherto existing determinations of line widths have been, however, quite unsatisfactory. The reason is that in these studies Balmer line parameters were used [see Hanuschik (1989) and references therein] which are strongly broadened by radiative transfer and Thomson scattering in addition to kinematic broadening. Because the question of geometry and kinematics is of crucial importance for understanding the $\mathrm{Be}$ phenomenon, I have started a new study, using measurements of the Fe II $\lambda 5317$ line. This line shows almost optically thin emission and is broadened primarily by kinematics.

\section{Fe II line width}

In Fig. 1 (top), total width of this line, $\Delta v_{\text {tot }}$, is plotted vs. stellar $v_{*} \sin i$ (taken from Slettebak 1982). There exists an unambiguous correlation,

$$
0.5 \Delta v_{\text {tot }} \approx 1.2 v_{*} \sin i \text {. }
$$

This correlation proves axisymmetry of the envelope and gravitationally dominated gas motion (rotation or infall). Observations of shell lines can help to further differentiate between these two cases. Shell line widths essentially indicate the range of radial velocities, $v_{\text {rad }}$, with disappearing derivative $\mathrm{d} v_{\text {rad }} / \mathrm{d} x$ in front of the star ( $x$ being the coordinate towards the observer). For a Keplerian disk, this spread is only $\approx-\Delta v_{\mathrm{D}} \ldots+\Delta v_{\mathrm{D}}$ or about 4 $\mathrm{km} \mathrm{s}^{-1}$ in case of iron at $10^{4} \mathrm{~K}$. For gravitational infall, this spread is larger, and the line becomes strongly redshifted. Figure 1 (bottom) shows the Fe II

* Now at: Astronomical Institute, University of Tübingen, D-72076 Tübingen, Germany 

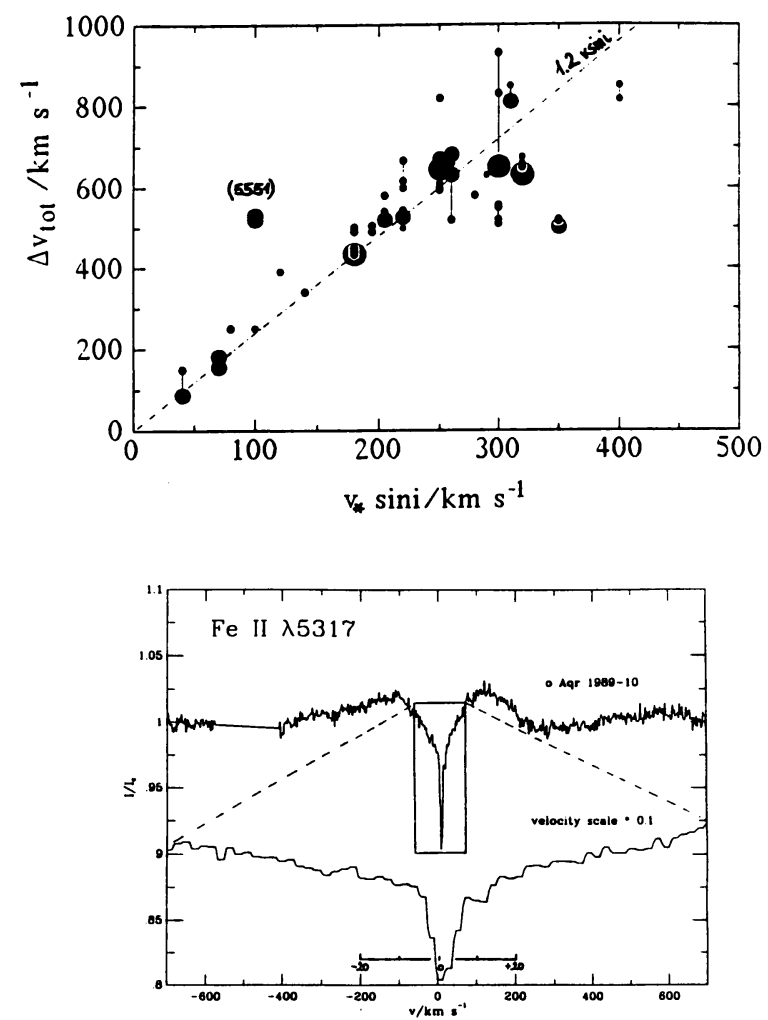

Fig. 1. Top: Total width $\Delta v_{\text {tot }}$ vs. $v_{*} \sin i$ for 27 stars. Size of symbols corresponds to number of measurements. Bottom: Fe II $\lambda 5317$ in $o$ Aqr.

shell line in o Aqr in Oct. 1989: it is symmetric around zero, and unresolved at resolution $6 \mathrm{~km} \mathrm{~s}^{-1}$ ! This line is clearly originating in a perfect Keplerian disk, putting a very strict upper limit on radial motions (less than about 3 $\mathrm{km} \mathrm{s}^{-1}$ ), thus contradicting any model with wind-like outflow.

Other stars (e.g. $48 \mathrm{Lib}, \zeta$ Tau) show broader, but asymmetric shell lines which are cyclically variable. These are presumably due to perturbed disks, cf. Hanuschik et al. and Hummel \& Hanuschik (these proceedings).

\section{Acknowledgements}

I would like to thank Wolfgang Hummel for providing unpublished measurements and for valuable discussions.

\section{References}

Hanuschik R.W.: 1989, Ap\&SS 161, 61.

Slettebak, A.: 1982, ApJS 50, 55. 